\title{
Computational simulation for analysis and synthesis of impact resilient structure
}

\begin{abstract}
Impact resilient structures are of great interest in many engineering applications varying from civil, land vehicle, aircraft and space structures, to mention a few examples. To design such structure, one has to resort fundamental principles and take into account progress in analytical and computational approaches as well as in material science and technology. With such perspectives, this work looks at a generic beam and plate structure subject to impact loading and carry out analysis and numerical simulation. The first objective of the work is to develop a computational algorithm to analyze flat plate as a generic structure subjected to impact loading for numerical simulation and parametric study. The analysis will be based on dynamic response analysis. Consideration is given to the elastic-plastic region. The second objective is to utilize the computational algorithm for direct numerical simulation, and as a parallel scheme, commercial off-the shelf numerical code is utilized for parametric study, optimization and synthesis. Through such analysis and numerical simulation, effort is devoted to arrive at an optimum configuration in terms of loading, structural dimensions, material properties and composite lay-up, among others. Results will be discussed in view of practical applications.
\end{abstract}

Keyword: Impact analysis; Composites; Engineering analysis; Engineering design; Finite element analysis; Structural analysis; Structural dynamics 